\title{
Determination of Isolated Salmonella Bacteria from Infected Animal Husbandry of Malayer City with using Molecular Methods
}

\author{
Alireza Khakzad ${ }^{1} \&$ Fatemeh Keshavarzi $^{2}$ \\ ${ }^{1}$ Department Of Biology, Sanandaj Branch, Islamic Azad University, Sanandaj, Iran \\ 2 Department Of Biology, Sanandaj Branch, Islamic Azad University, Sanandaj, Iran \\ Correspondence: Fatemeh Keshavarzi, Department Of Biology, Sanandaj Branch, Islamic Azad University, \\ Sanandaj, Iran. E-mail: alireza.khakzad@chmail.ir
}

Received: December 30, 2016

Accepted: January 16, 2017

Online Published: March 8, 2017

doi:10.5539/jmbr.v7n1p34

URL: https://doi.org/10.5539/jmbr.v7n1p34

\begin{abstract}
Salmonella species are gram negative bacteria and members of Enterobacteriaceae family. It has a rod-shaped appearance; it is catalase positive, oxidase negative, non-spore. Salmonella classified into two species, Salmonella Enterica and Salmonella Bangori. Salmonella is now one of the main reasons of diarrhea and vomiting in humans in many countries and especially in industrialized. In a study in Japan 164 Salmonella digestions were collected during 2006 to 2008 which 81 digestions were Salmonella Infantis. Salmonella-specific characteristics are studied in the two phenotype and genotype methods. In this research, with using genotype methods based on PCR, genetic diversity was evaluated; this PCR includes rep-PCR based on repetitive sequence elements (method was done by the use of three primers ERIC, REP and BOX). Studied showed most isolated strains were relevant to Salmonella Enteritidis and dendorogram study showed that the bacteria were grouped in one cluster in dendrogram that all 37 strains were put in a large cluster of Salmonella's type which is divided into two clusters: Salmonella Enterica and Bongori. The results also in this experiment reflect the efficiency of rep-PCR method by using three ERIC, REP and BOX primers.
\end{abstract}

Keywords: Salmonella, phenotype, genotype, rep-PCR, primer

\section{Introduction}

Salmonella was observed for the first time in 1880 by Carl Ebert in spleen samples of patients with typhoid fever. Four years later, George Theodore proliferate this pathogen in culture (Anderson et al., 2010). A year after that, the doctor called Smith, medical research scientists, achieved a novel discovery what later became known as Salmonella Enterica. At that time, Smith was working as an assistant in the Department of Veterinary Research Laboratory of the US Department of Agriculture. This department was under supervision of Daniel Elmer Salmon, a veterinary pathologist who later because of glory of this pathogen discovery by Daniel Salmon called Salmonella to this bacteria (Asadpour et al., 2013). This genus of gram-negative bacteria such as the other Enterobacteriaceae family members has a rod-shaped appearance, positive catalase, negative oxidase, non-spore. These microorganisms have a diameter of 0.7 to 1.5 micrometers and a length of 2 to 5 and their main habitat is the digestive system of humans and animals. They are optional anaerobic bacteria and movable and very high compatibility of these genus members allows them to infect a wide range of their hosts.As a reference method, the serotypes determination is a useful technique in source documents research. However, the serotypes determination in isolated instances needs to present to reference laboratories which have specific antibodies and employ trained personnel; serotypes determination can take several days to several weeks (EFSA, 2010). This led to accept some methods based on nucleic acid that will cause rapid determination of Salmonella strains to trace the source. These methods are especially useful in the animal husbandry and poultry industry. However, determination the frequency of Salmonella serotypes in infected animals have not been conducted in Malayer by molecular methods (Farzan et al., 2006). Also, salmonellosis is a common gastrointestinal disease in the world. This disease caused by different Salmonella bacteria serotypes. There are concerns about contamination of poultry and its products to this microorganism. According to carcasses of poultry are proposed as an important source of infection to this disease, determination of the frequency and extent of contamination and serotype diagnosis can be considered a criteria for evaluating the health status of slaughterhouses and health potential hazards determination for consumers (Refereed, 2003). Pathogenesis of this disease has been observed frequently so that these microorganisms may be moved vertically or horizontally in aviculture and be alive 
in various processing. Many basic studies show that Salmonella's carting rate after slaughter in broiler chicken carcasses is different in the different continents and changes from 7/36\% in Australia to $7 / 15$ in Europe and 0\% / 21\% in America (Ghadrdan, Ghorbanpour \& Khazaeil, 2007). Bacteria prevalence results in slaughtered carcasses are associated with human salmonellosis and poultry products consumption among the people. The aim of this study can be expressed in several sections; it includes: Identification and determination of different salmonella serotypes frequency, obtaining the most common serotypes of Salmonella in livestock of Malayer city, determination of relationship between the serotypes frequency with animal pregnancy status, control and effective epidemiological monitoring to identify potential sources of infection with Salmonella serotypes, determination the relationship between the serotypes with livestocks age in Malayer city and serotype's frequency relationship with antibiotics usage in 3 months before in livestocks.

\section{Materials and Equipment}

In this study, we used different materials to catch results which are mentioned in Table 1 in details. In this research, special equipments for laboratory works fulfillment was used that their names and characteristics are mentioned in Table 2.

Table 1. Used materials in research

\begin{tabular}{ll}
\hline Used materials & Used materials \\
\hline Taq enzyme polymerase & TSI \\
primers & MRVP \\
DNA extraction kit & MgCl2 \\
Simmon Citrate Agar growth medium & Buffer TBE \\
Urea growth medium & Distilled water \\
SS agar growth medium & Agarose \\
F selenite growth medium & phenol \\
Alcohol & formaldehyde \\
\hline
\end{tabular}

Table 2. Used equipment

\begin{tabular}{ll}
\hline Equipment model & Equipment name \\
\hline F2000 & Autoclave \\
CamSpec M501 Single Beam UV/Visible & Spectrophotometer \\
Eutech instruments pH 510 & pH metr \\
AND & Digital balance \\
Labtron & Incubator \\
HD & Oven \\
DWJ & Water distillation \\
Hettich & Centrifuges \\
Nikon & Microscope \\
Northen & Thermocycler \\
TM52A & Hood \\
\hline
\end{tabular}

\section{Sampling Way and Samples Cultivation}

In this study conducted in February of 2015, in some animal husbandry in Malayer, totally 50 sheep in 6 months to 1 year age ranges were sampled. After attending in appropriate animal husbandry, each livestock's information (such as name, gender, age, race, etc.) was recorded in special form. Then, stool sampling was conducted. In sheep selection time for the sampling, the following cases were considered, since sampling was conducted in two phases and intervals of 5 days, rather assurance of livestock remaining in animal husbandry during appropriate weeks was given. Also, in this period, livestock under studied should not have to exit from animal husbandry. Sampling from fresh stool of each sheep was conducted. Samples culture in special growth media of Shigella- Salmonella: to isolate the bacteria Salmonella from other microorganisms and bacteria exist in the taken stool cultures, Salmonella-Shigella (SS) agar growth media was used so that after removing the Selenite broth from the oven, the contents of each universal tube was cultured in one simultaneous loop on brilliant green agar and SalmonellaShigella agar in four regional approach and these environments for 24 to 48 hours at $37^{\circ} \mathrm{C}$ in oven were kept. 
Isolation and identification of Salmonella: suspected colonies to spread salmonella were provided and colored by Gram staining. Oxidase negative and gram-negative salmonella digestions were identified by conventional biochemical methods. Finally, samples were identified based on different microbiology tests. In this study, biochemical tests such as transport on the urea environment - Simon citrate agar, Triple sugar iron agar (TSI) and MRVP are used. To confirm diagnosis at this stage, serotyping test with using $\mathrm{O}$ and $\mathrm{H}$ antiserum according to the manufacturer's instructions (Statern Serum Institute, Copenhagen Denmark) on the slide agglutination was performed.

DNA Extraction: DNA was extracted by phenol-chloroform. Thus, Salmonella purified samples after 24 hour culture on the Luria broth culture medium from the bacterial suspension was prepared and the exists sediment based indicated sizes in the Table 3 lysis buffer and proteinase K and SDS were added and this suspension at $60^{\circ}$ $\mathrm{C}$ for one hour were incubated. At the next step, the solution of phenol-chloroform-isoamyl with the ratio of 1-2425 was used for protein separation and at the end. To purify DNA, cold ethanol 90 and 70 percent was added to supernatant phase of solution and purified DNA in 75 micro liters of distilled water was dissolved. To determine concentration and purity of extracted DNA, the obtained product was loaded on agarose gel. Bands were examined. The ratio of light absorbance at $260 \mathrm{~nm}$ to $280 \mathrm{~nm}$ was also measured by spectrophotometry.

Table 3. Amount and type of used materials for suspension providing from extracted DNA from Salmonella purified samples

\begin{tabular}{cc}
\hline Amount of used materials(micro-liter) & Materials used in DNA extracted suspension \\
\hline 620 & Liz Buffer \\
13 & SDS $2 \%$ \\
3 & Proteinse K \\
\hline
\end{tabular}

\section{Determination of Genetic Diversity using Molecular Techniques}

There are various methods to determine the genetic diversity of the species and different strains that one of them used in this study. To study the genetic diversity of pathogenic bacteria from rep-PCR is used. In this method, three sets of primers consisting of REP, ERIC and BOX are used. This method uses primers which target repetitive non-coding sequences that spread the whole genome of bacteria and fungi. To do the ERIC-PCR method, DNA samples belong to different strains was used with using of primers below:

ERIC1R 5'-ATGTAAGCTCCTGGGGATTCAC-3'

ERIC2F 5'-AAGTAAGTGACTGGGGTGAGCG-3'

To do the REP-PCR method, REP1R-I and Rep2F-I primers were used, their sequences are mentioned below:

\section{REP1R-I (5'- IIIICGICGICATCIGGC-3)}

REP2F-I (5'-ICGICTTATCIGGCCTAC-3)

Fingerprint with using of BOX conserved sequences based on their types has a sequence near 45 to 59 pairs that is performed for a bacteria such salmonella. In this method, BOX AIR primer with following sequence is used.

\section{5'-CTACGGCAAGGCGACGCTGACG -3'}

PCR test was done by Eppendorf thermocycler and in a volume of 20 micro-liters and the amount of contained material in the Table 4 is inserted. ERIC-PCR process in the Table 5 is inserted.

Table 4. Amount and type of used material in ERIC-PCR

\begin{tabular}{ccc}
\hline Amount of used materials(micro-liter) & Materials used in ERIC PCR \\
\hline 12 & Deionized water \\
2 & $10 X$ BufferPCR \\
1 & $\mathrm{MgCl}_{2} 5 \mathrm{mM}$ \\
1 & $\mathrm{dNTPs} 10 \mathrm{mM}$ \\
1 & Each primer with 20 pmol \\
1 & Taq DNA polymerase \\
\hline
\end{tabular}


Table 5. ERIC-PCR method

\begin{tabular}{cccc}
\hline Cycle number & Time $(\min )$ & Temperature (Celsius) & Steps/conditions \\
\hline 1 & 4 & 94 & Primery denaturation \\
35 & 1 & 94 & Denaturation \\
35 & 1 & 52 & Primers binding \\
35 & 8 & 65 & Primer synthesis \\
1 & 15 & 65 & Last synthesis \\
\hline
\end{tabular}

Electrophoresis gel: PCR products on agarose gel 1\% electrophoresis with voltage 70 for an hour were electerophoresed and finally the gel was photographed. ERIC-PCR schemas were compared with visualizing and their placements on the gel according to their molecular weight were calculated and molecular markers and bands counting for each sample were performed. Comparing PCR schema on the website of Insilico.ehu.es was performed, so that a matrix of zero and one was provided that shows the presence or absence of a bond and on the website were recorded and the basic of calculating genetic patterns by the websites were observing their placements in gel according to their molecular weight and markers and counting the bands. Power of PCR method discrimination was conducted by the Simpson's Index of Diversity equation (1) (Schwarz \& Kehrenberg \& Walsh, 2001).

$$
D=1-\frac{1}{N(N-1)} \sum_{j=1}^{s} n_{j}\left(n_{j}-1\right)
$$

$\mathrm{N}$ is the whole number of studied strains for ERIC-PCR method. $\mathrm{S}$ is the number of calculated genetic types and $\mathrm{n}_{\mathrm{j}}$ is the number of strains count belong to $\mathrm{j}$ type.

\section{Statistical Analysis of Data}

Used indicators (BOX and ERIC, rep) are dual-mode. Therefore, the presence or absence of bands is shown with one and zero respectively. Coefficient of similarity is between the studied isolates and genotypes grouping with using of NTSYS-PC (Ver. 2.02) software and UPGMA methods carried out. Resultant dendrograms were compared. Correlation between cophenetic and similarity matrix was used as a criterion to evaluate the goodness of clustering fitness. Also, to investigate the relationship between similarity matrices Mantel test was used.

\section{Tests Method}

$2 \mathrm{~g}$ of each stool was put in the F 10 enrichment medium for integration. Finally, samples were heating in the oven for 6 hours at 37 degrees Celsius. Standard method of salmonellosis diagnosis is the stool bacterial culture (Lester $\&$ Vetrowidenhouse, 2003). In this study mentioned method was used. Salmonella Shigella agar culture samples (SS): considered samples culture which was in oven before, was on the Salmonella -Shigella agar medium growth. In Figure1, samples culture on brilliant green agar and Salmonella -Shigella agar shows in four- regional method.
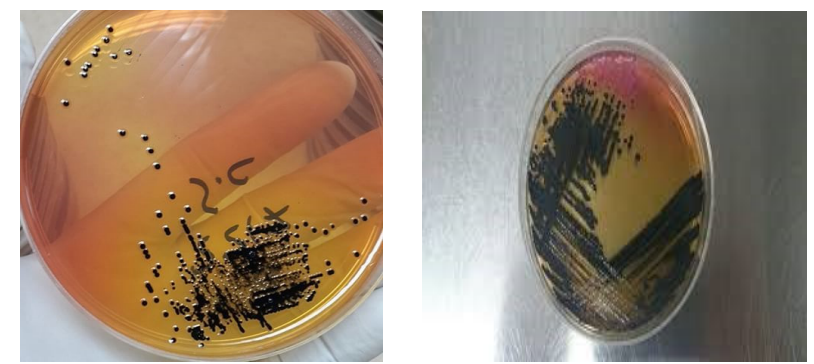

Figure 1. Exist cultured salmonella sample in the stool sample in SS agar growth medium

\subsection{Isolation and Salmonella Identification}

First, to isolate salmonella from other microorganisms and ensuring from salmonella existence in SS agar medium culture, staining method was used for salmonella isolation as a gram-negative. Some tests was used that include urea environment - Simon citrate agar, Triple segar iron agar, MRVP and negative oxidase. Serotyping test with using $\mathrm{O}$ and $\mathrm{H}$ antiserum on the agglutination slide was used for confirmation. In this study, 50 sheep were sampled in two phases with intervals, 17 of 50 sheep was infected to salmonella which had one year and belong to three animal husbandries in Malayer city .Also, based on study results report, serotype of submitted Salmonella Enteritidis digestion was determined . In this study, the Salmonella infection in livestock was $34 \%$. The differences between the present results with other studies must be reviewed, because many changes in results can be obtained 
due to climatic and management differences. Totally, it is said that determination of salmonella defecation amount from stool with lack of clinical symptoms is very hard and test precision and precise results are dependant to geographical location, season and bacteria isolation method, too. Antiserum results showed that 37 digestions from whole isolated serovars were relevant to Salmonella Enterica, subspecies of Salmonella Enteritidis, with a frequency of $80 \%$. Therefore, this serotype strains were selected and studied more by molecular methods.

\subsection{DNA Extraction from Isolated Salmonella Samples}

Bacteria DNA was extracted with phenol-chloroform DNA extraction and washed with ethanol $70 \%$ and $90 \%$ that was loaded on agarose gel for assurance. Figure 2 is the extracted DNA sample.

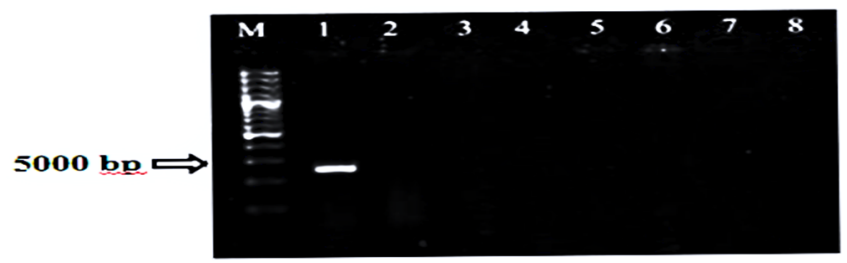

Figure 2. Extracted DNA from bacteria and its comparison with ladder

\subsection{Determination of Genetic Types}

After isolation and purification, PCR reactions were performed to identify the considered isolates. At this stage, for isolates identification with using rep- PCR, three different primers REP, ERIC and BOX was done. One of important works for serotype and samples genetic diversity identification is PCR using that with specific primers, specific sequences transcript a lot. It is used for identification and detection of bacteria serotype. Profiles can be analyzed with band number and locations of amplified DNA checking. Number of observed profiles for each primers based on polymorphs of profiles is in the base pairs range of each primers. For example, for ERIC primer, three polymorphic profiles and with same number of base pairs was created in base pairs range. Also, the reason of two profile b1 existence is that they are polymorphism. In order to determine the reproducibility, PCR fingerprinting or rep- PCR method was used that repetitive samples were analyzed and electrophoresis was performed in agarose gel, at place, each samples running profile was measured with the side sample. Of course, some bands have dissimilar patterns of similar samples and the difference in band severity was sometimes observed. A lot of bands were weak and showed that there are not repetitive sequences. Then it could not be considered. Many bands were higher than 1500 base pairs that make their analysis hard. Composed bands between 140 and 1500 base pairs were analyzed in better forms, then, to have precise results and better detection, in this method, the some primers matching relevant with fingerprint of rep-PCR repetitive sequence was used and with using three ERIC, REP and BOX primers were performed. Except weakness or lack of one or more DNA bands, many of repPCR bands were checkable. Checking genomic profile of isolates on $1.5 \%$ agarose gel were performed. All repetitive and clear bands in 150 to 1700 base pairs were scored based on zero and one code. Totally, from three ERIC, REP, BOX bands, 33 polymorph bands resulted which would be explained in below more. In this study, DNA fragments resultant from REP-PCR made from some stable and repetitive bands which is observable. As shown in Figure 3, with ERIC_PCR method, digestions were divided to three main profiles E1, E2, E3. Profiles were determined based on differences in number and size of bands. In summary, different bacteria digestions resultant from ERIC-PCR primer had 11 to 14 bands that in bands or fragments, near 100 or 1450 base pairs were observed. The approximate size of observed bands in this study in E1 profile includes 150, 250, 370, 400, 490, $680,720,810,930,1050,1200,1400$ base pairs. E2 profiles showed bands near E1 profiles that all these bands were observed in E3 profile. As it is clear, differences of $\mathrm{E} 2$ with $\mathrm{E} 1$ profile is added band near 600 base pairs. E3 profile shows the presence of a band with 280 base pairs that can't be seen in other profiles. Also, band existence near 250,680,810 in outgroup serovars is observable. Also, band characteristics of E1 profile found in 110 digestions relevant to salmonella enteritidis separated from foodstuffs. E2 and E3 profile bands were from RTCC standard strains type. Similarity between each E1,E2, E3 clusters samples was 84,89, 79 percent respectively. Also, E1 and E2 had 91 percent similarity. Serotype checking showed that E1 profile includes 4 clusters and 11 digestions, E2 includes 2 clusters and 9 digestions and E3 includes 1 digestion. In summary, in 14 different bacteria digestions from salmonella enteritidis near 11 to 14 bands were observed by ERIC-PCR. Comparison and molecular analysis were performed on bands resultant from genome of separated strains with other standard strains, they were close to the specie in the genetic sequences view and were separated from hosts and typing were carried out in other studies. It was performed to analysis the electrophoresis in Figures 3, Figure 4 and Figure 5. Because, most of the separated serovars from livestock were relevant to salmonella enteric, subspecies of salmonella 
enteritidis. For this reason, as a outgroup or control group four serovars were used: salmonella panama SP, salmonella berendi S.B, salmonella agona SA, salmonella typhimurium ST.

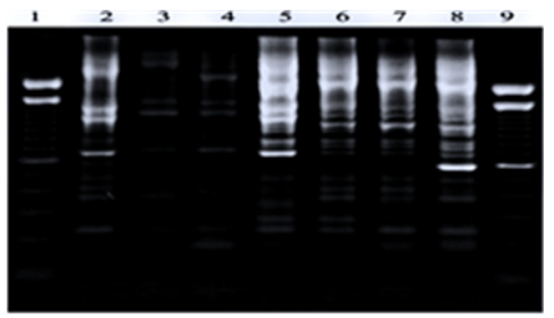

Figure 3. Different patterns resultant from ERIC-PCR strains of salmonella enteritidis on agarose electrophores gel $1.5 \%$ with staining or ethidium bromide

Results of checking this primer in three different profiles E1, E2, E3 were clustered. Column 1 ,9 molecular marker of 100 nucleotides bases, size indicator, salmonella panama SP, salmonella berendi S.B, salmonella agona SA, salmonella typhimurium ST. With composition of I -REP1R4 and I-REP2 primers, some different profiles were determined which were clustered in three different profiles R1, R2, R3. PCR optimization with using of standard strains of salmonella enteritidis lead to amplification of a fragment of 250 base pairs from bacteria genome. PCR results checking on accumulated samples showed mentioned fragment formed in 17 cases in 50 cultured stool samples. Results of this primer checking are in Figure 4 that 8 to 11 bands show in 140 to 1340 fragments. R1 profile showed all the patterns characteristics. Conserved bands found in near 140,580, 710, 785,1060, 1300 base pairs in all salmonella enteritidis digestions. R2 and R3 profile showed similar bands toR1 band but with some added. Characteristic checking of R2 profile showed the existence of a weak band near 140 base pairs with two added fragment from 330 to 500 base pairs. Characteristic checking of R3 profile, showed two added bands rather than R1, near 180 and 360 base pairs. Comparison of three profiles showed 77.4\% similarity. R1 and R3 profiles with forming one cluster only had $83 \%$ similarity. Detected bands in R1 profile showed high similarity in digestion bands of typhimurium but with other serovars including salmonella panama, salmonella berendi, salmonella agona, formed one different cluster with similarity below the 70 percent. Most of the digestions in this test was relevant to R1 profile and while characteristic of R2 profile is found only in one of the found digestions in (RTCC) collection and this digestion is detected in broiler.

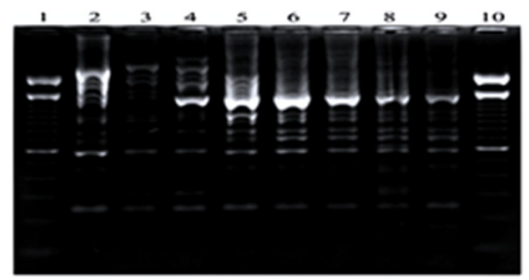

Figure 4. Checking relevant to studied PCR fragments in salmonella serotype with using of REP_PCR primers in agarose electrophoresis gel $1.5 \%$ with staining or ethidium bromide

Results of checking this primer in three different profiles R1, R2, R3 were grouped. Size indicator (sink 1,10), salmonella panama (SP), salmonella berendi (SB), salmonella agona (SA), salmonella thyphi murium(ST). Electrophoresis gel checking BOX-PCR with BOXA1R primer was showed in three different profiles BOX1, BOX2, BOX3 (Figure 5). Product size was near 140 base pairs to 1230 . Indicator profile was 8 or 9 bands in sink length. All salmonella digestions were near 160, 225, 430, 520, 645, 880, 1260 base pairs, considered fragment near 645 base pairs was relevant to another serovar. B1 Profile characteristic results were recognized in fragments near 140,245, 380, 520, 440, 645, 880, 1100, 1260 base pairs. This checking results showed that difference of B1, B2 profile is a fragment near 750 base pairs and lack of a fragment near 1080 base pairs. Checking determined bands of B3 profile shows that except a fragment with 380 base pairs, the same observed fragments were observed in B1. All digested salmonella belongs to B1 profile and B2 characteristic showed only an added digestion. PCR showed $85.6 \%$ similarity in strain detection of salmonella entertidis with using BOX primer. B1 and B3 profile with forming one cluster showed only $94.1 \%$ similarity. Salmonella and other serovar showed less than $70 \%$ similarity of band pattern. 


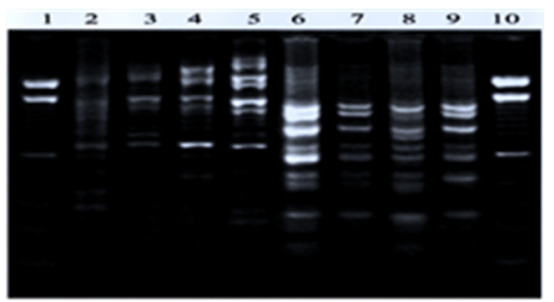

Figure 5. Checking relevant to studied PCR fragments in salmonella serotype with using of BOX_PCR primers in agarose electrophoresis gel $1.5 \%$ with staining or ethidium bromide

\subsection{Resultant Dendrogram from Primer Pattern}

Resultant DNA band or fingerprint from REP-PCR type amplification was checked and the existence of band in gel and absence of each bands in data profiles defined score "1" and " 0 ", respectively. Dendrogram results were drawn with data integration in Numerical Taxonomy System, (NTsys 2.0) software, a software to calculate similarity index with simple genetic matching. Comparison between strains bands and data resulted from dendrogram and is shown in Figure 6. Dendrogran cluster analysis was checked in salmonella enteric based on REP_PCR, composition of three different primers REP, ERIC, BOX. Genotype patterns were formed by these three primers showed 90 percent similarity. As most strains relevant to a serotype were in a close clads, but in similarity level, all the strains relevant to a serotype separated of each other. Totally, from different digested isolates and checking band and data pattern result from 37 strains were detected which most of them belong to the Salmonella Enterica. Except typhimirium, one number (isolated 37) which was a number originated from human and Bongori strain with one number (isolated 34) detected from RTCC standard type, too. Digestion types 2, 7 of poultry and isolated 2, 3, 7, 8, 9 in human samples of foodstuffs and fork were determined. Isolated 13, 24, 31 in human and foodstuffs were observed.

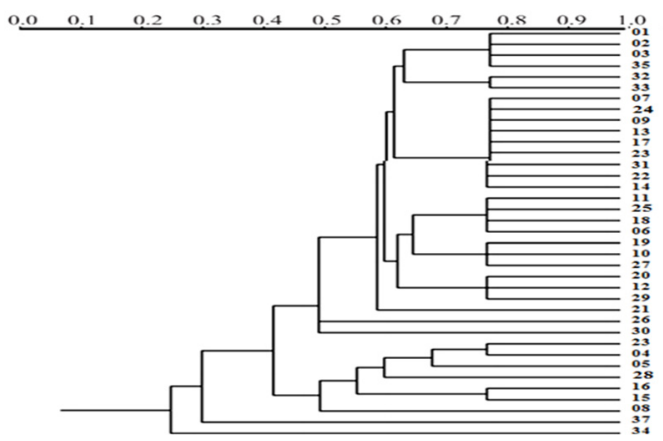

Figure 6. Results of isolated similarity with using of dendrogram chart (drawed with NTsys 2.0) software

Data determination is on vertical axis and numbers are on horizontal axis. Determination of similarity percent between profiles with checking Jaccard coefficient and dendrogram had been drawn with using UPGMA averaging method. Data related to similarity were studied and bacteria were grouped in one main cluster in dendrogram that all 37 strains were in a big cluster. Main cluster were relevant to salmonella type that divide to two Salmonella Enterica and bongori. In this dendrogram, one strain (34) is salmonella bongori according to evidence and others are Salmonella Enterica that strain 37 is relevant $t$ salmonella typhimurium and other strains are relevant to salmonella enteritidis with different serovars.

\section{Discussion and Conclusion}

This study is a summary of research. This research aimed to determine salmonella bacteria serotypes from infected livestock of Malayer city with molecular methods. To do this research, a cross-sectional study between October 2015 and August 2016 were performed. This study's main goal was determination of common salmonella serotypes proportion in collected samples from infected farms.In present study, salmonella infection amount in livestock were 34 percent. Results of antiserum showed that from digested serovars from livestock, 35 digestions were relevant to Salmonella Enterica which next molecular study determined this subtype frequency as salmonella enteritidis with 80 percent frequency. Totally, in this study, four repetitive elements of polymerase chain reaction 
(primers ERIC1R ،ERIC2 ‘REP1R ،REP2I, BOXA1R) were studied for salmonella determination in serotype level. Checking number, only all fingerprints and clearance of fingerprint bands, ERIC primer set were used for these tests. For this primer, repetitivenece on lysate of selected salmonella serotypes in PCR tested and different PCR were performed for this work. All counted bands in digestions were 86. Isolates 11 and 15 with 9 bands had most amplified bands number and isolates 31 and 6 had lowest amplified bands by considered primer. Also, fingerprint performed on more than 37 serotypes and some other isolates which were checkable with serotyping. Fingerprint was performed to evaluate its detection and recognition of other primers. At last, all species were fingerprinted by ERIC, BOX, REP primer set. At last, different species were recognized to different serotypes that performance results showed rep-PCR balance in tested serotype types. More than this, test showed that ERIC primer have a high performance in salmonella serotype detection but one serotype can't be checked only with ERIC and using other primer relevant to repetitive fingerprint sequence can help in better detection of serotypes. This repetitive fingerprint sequence includes REP and Box. Most study shows that salmonella typhi is a concerning pathogen in developmental countries especially Asia (European Food Safety Authority, 2012). In present study, existence of infection in livestock in a small scale of one city presents same infection in a more wide range that indubitable, it must be considered to health-organization.

\section{References}

Anderson, P. N., Hume, M. E., Byrd, J. A., Hernandez, C., Stevens, S. M., Stringfellow, K., \& Caldwell, D. J. (2010). Evaluation of repetitive extragenic palindromic-polymerase chain reaction and denatured gradient gel electrophoresis in identifying Salmonella serotypes isolated from processed turkeys. Poultry science, 89(6), 1293-1300. http://dx.doi.org/10.3382/ps.2009-00389

Asadpour, Y., Mohammadi, M., Pourbakhsh, S. A., \& Rasa, M. (2013). Isolation, serotyping and antibiotic resistance of Salmonella isolated from chicken carcasses in Guilan province. BMC veterinary research, 9(2), $5-13$.

EFSA, C. (2010). Trends and sources of zoonoses and zoonotic agents and food-borne outbreaks in the European Union in 2008. EFSA, 8(2), 1-370.

European Food Safety Authority. (2012). Multi-country outbreak of Salmonella Stanley infections Update. EFSA Journal, 10(9), 2892-2893. http://dx.doi.org/10.2903/j.efsa.2012.2893

Farzan, A., Friendship, R. M., Dewey, C. E., Warriner, K., Poppe, C., \& Klotins, K. (2006). Prevalence of Salmonella spp. on Canadian pig farms using liquid or dry-feeding. Preventive Veterinary Medicine, 73(4), 241-254. http://dx.doi.org/10.1016/j.prevetmed.2005.09.003

Ghadrdan, M. A., Ghorbanpour, M., \& Khazaeil, K. (2007). Survey on salmonella infection rate in horses of some horse riding clubs in Ahvaz. American Journal of Drug Discovery and Development, 3(3), 181-187. http://dx.doi.org/10.3923/ajdd.2013.181.187

Lester, G. D., \& Vetrowidenhouse, T. S. (2003). Salmonellosis. In: Robinson, N.E. (Ed). Current therapy in Equine Medicine. 5th ed. Saunders, United State of Americ.

Refereed, N. (2003). Pre-harvest food safety diagnostics for Salmonella serovars. Part 2: Molecular diagnostics. Journal of Swine Health and Production, 11(3), 140-141. Retrieved from https://www.aasv.org/shap/issues/ $\mathrm{v} 11 \mathrm{n} 3 / \mathrm{v} 11 \mathrm{n} 3 \mathrm{p} 141$

Schwarz, S., Kehrenberg, C., \& Walsh, T. R. (2001). Use of antimicrobial agents in veterinary medicine and food animal production. Int. J. Antimicrob. Agents, 17(2), 431-437.

\section{Copyrights}

Copyright for this article is retained by the author(s), with first publication rights granted to the journal.

This is an open-access article distributed under the terms and conditions of the Creative Commons Attribution license (http://creativecommons.org/licenses/by/4.0/). 